\title{
On the phase structure of two-dimensional generalized Yang-Mills theories
}

\author{
Masoud Alimohammadi ${ }^{a}$ 円 \& Mohammad Khorrami $^{b}$ 2 \\ ${ }^{a}$ Physics Department, University of Teheran, North Karegar Ave., \\ Tehran, Iran \\ ${ }^{b}$ Institute for Advanced Studies in Basic Sciences, \\ P. O. Box 159, Zanjan 45195, Iran.
}

\begin{abstract}
The phase structure of the generalized Yang-Mills theories is studied, and it is shown that almost always, it is of the third order. As a specific example, it is shown that all of the models with the interaction $\sum_{j}\left(n_{j}-\right.$ $j+N)^{2 k}$ exhibit third order phase transition. $\left(n_{j}\right.$ is the length of the $j$-th row of the Yang tableau corresponding to $\mathrm{U}(N)$.) The special cases where the transition is not of the third order are also considered and, as a specific example, it is shown that the model $\sum_{j}\left(n_{j}-j+N\right)^{2}+g \sum_{j}\left(n_{j}-j+N\right)^{4}$ exhibits a third order phase transition, except for $g=27 \pi^{2} / 256$, where the order of the transition is $5 / 2$.
\end{abstract}

PACS numbers: 11.10.Kk, 11.15.Pg, 11.15.Tk

Keywords: large-N, phase transition, generalized Yang-Mills, eigenvalue density function

${ }^{1}$ e-mail:alimohmd@theory.ipm.ac.ir

2e-mail:mamwad@iasbs.ac.ir 


\section{Introduction}

Recent works by many authors suggest that the pure Yang-Mills theory in two dimensions is equivalent to a string theory [1-7]. The reason to investigate such a simple theory in such great detail is partly to shed light on the strongcoupling limit of pure QCD in four dimensions. The string picture of the twodimensional Yang-Mills $\left(\mathrm{YM}_{2}\right)$ is also interesting on its own, as an example for nonperturbative analysis of a quantum field theory.

The starting point for making the correspondence of $\mathrm{YM}_{2}$ with string theory is the study the large $-N$ limit of $\mathrm{YM}_{2}$. For example, as it is shown in [1], [3], and [1], a gauge theory based on $\mathrm{SU}(N)$ is split at large $N$ into two copies of a chiral theory, which encapsulate the geometry of the string maps. The chiral theory associated to the Yang-Mills theory on a two-manifold $\mathcal{M}$ is a summation over maps from the two-dimensional world sheet (of arbitrary genus) to the manifold $\mathcal{M}$. This leads to a $1 / N$ expansion for the partition function and observables that is convergent for all of the values of area $\times$ coupling constant on the target space $\mathcal{M}$, if the genus is one or greater.

It is well known that $\mathrm{YM}_{2}$ is defined by the Lagrangian $\operatorname{tr}\left(F^{2} / 4\right)$ on a Riemann surface, where $F$ is the field-strength tensor. This theory is equivalent to the so-called $B-F$ theory, characterized by the Lagrangian $i \operatorname{tr}(B F)+\operatorname{tr}\left(B^{2}\right)$, integrated over the auxiliary field $B$. This theory is essentially characterized by two important properties: the lack of propagating degrees of freedom, and invariance under area-preserving diffeomorphisms. This theory, however, is not unique in possessing these two characteristics. These properties are also shared by a large class of theories, called the generalized two-dimensional Yang-Mills $\left(\mathrm{gYM}_{2}\right)$ theories. These theories are defined by replacing the term $\operatorname{tr}\left(B^{2}\right)$ in the $\mathrm{YM}_{2}$ Lagrangian by an arbitrary class function of $B$ [8].

Many of the physical features of $\mathrm{gYM}_{2}$ have been studied. Several aspects of the partition function of these theories has been discussed in [8-10]. The generating functional of the field strength for arbitrary two-dimensional orientable and non-orientable surfaces has been calculated in 11 (and in 12, 13 for the $\mathrm{YM}_{2}$ 's). In all of these calculations, the solutions appear as some infinite summations over the irreducible representations of the gauge group $\mathrm{U}(N)$ (or $\mathrm{SU}(N)$ ). In the large $-N$ limit, however, these summations are replaced by suitable path integrals over continuous parameters characterizing the Young tableaux, and a saddle-point analysis shows that the only significant representation is the classical one, which maximizes some effective action.

The continuous function characterizing the representation is a constrained function, as the length of the rows of the Yang tableau is nonincreasing. This means that the problem of minimizing the effective action is a constrained one, and in fact the constraint is a nonholonomic one. For small values of the surface area, the classical solution satisfies the constraint; for large values of the surface area, it does not, and the dominating representation is not the one which minimizes the effective action. This introduces a phase transition between these 
two regions, which has been studied in (for example) [14, 15] for $\mathrm{YM}_{2}$. This same problem has been studied for some special cases of gYM 2 's [16]. In [17], the density function $\rho$ has been obtained for an arbitrary $\mathrm{gYM}_{2}$ in the weakcoupling region. $\rho$ is essentially the density function of the length of the rows of the Young tableau, and by weak-field region it is meant that the surface area $A$ is less than some critical area $A_{c}$. Moreover, calculating $\rho$ in the strong region $A>A_{c}$ (near the critical point $A_{c}$ ), it is shown that the phase transition of the theory corresponding to the interaction $\sum_{j}\left(n_{j}-j+N\right)^{4}$ is of the third order, the same behavior seen for $\mathrm{YM}_{2}$. Finally in $[18$, the phase transition of $\sum_{j}\left(n_{j}-j+N\right)^{6}$ and $\sum_{j}\left(n_{j}-j+N\right)^{2}+g\left(n_{j}-j+N\right)^{4}$ (for $\left.g<<1\right)$ has been proved to be of the third order. In all of these works, the procedure of investigating the phase transition is based on the direct calculation of $\rho_{s}$ (the density function in the strong region $A>A_{c}$ ). The form of this function is complicated and obtaining a closed form for it, for arbitrary $\mathrm{gYM}_{2}$ 's, is not easy, even near the critical point.

In this paper we are going to investigate the order of the phase transition for a typical $\mathrm{gYM}_{2}$, by a different method. In this method, only the behavior of $\rho_{w}$ (the density function in the weak-coupling region) is needed, and in fact this behavior is needed only near the critical point. In this way, one finds the phase transition of almost all of $\mathrm{gYM}_{2}$ 's is of the third order. Nontypical $\mathrm{gYM}_{2}$ 's are also investigated and the criteria for a theory to be typical, that is its phase transition be of the third order are also studied. It is shown that if the second derivative of the weak-region density function is negative at its absolute maximum for $A=A_{c}$, and if its first derivative with respect to $A$ is positive at the same point, then the theory exhibits third order phase transition. It is further shown that theories with monomial interactions satisfy these criteria. It is also shown that if the second criterion is violated, then the order of transition will be an integer times 3 . Finally, a theory with quadratic as well as quartic interaction is analyzed and it is shown that it is typical except for a specific value of the coupling constant, where it exhibits a transition of the order $5 / 2$.

Let us investigate this nontypical behavior more closely. It was stated that if, at the critical point, the first derivative of the absolute maximum of the density function with respect to $A$ is positive, and the second derivative of the density function at its absolute maximum is negative, then the theory is typical, that is the order of the transition is 3 . What is known, is that at the critical point the absolute maximum of the density function is an increasing function of $A$. The reason is that for $A<A_{c}$, this absolute maximum is less than one, as the system is in the weak region. For $A>A_{c}$, this absolute maximum should be greater than one. So it should pass one at $A=A_{c}$. This shows that the first derivative of this absolute maximum is nonnegative, but it may be zero at $A=A_{c}$. Theories for which this derivative is zero exactly at $A=A_{c}$, are fine tuned. Normally, this derivative is positive. The second criterion for a theory to be typical can also be discussed the same way. At the absolute maximum of the density function, the first derivative of the density function is, of course, 
zero. The second derivative should be nonpositive. Theories for which this second derivative is zero exactly at the transition point, are fine tuned. Other theories are typical and exhibit a third order phase transition. In fact, in the example which shows a transition of the order (i.e. exponent) $5 / 2$, exactly this criterion is violated. The parameters of a theory are tuned so that at the critical point, the second derivative of the density function is zero, and the first nonzero derivative of that function is the fourth derivative.

The scheme of the paper is the following. In section 2, after a review of $\mathrm{gYM}_{2}$ 's, the criteria for the existence of the third order phase transition is studied and the order of the transition for nontypical theories is also studied. In section 3, it is shown that theories with monomial interaction are typical. In section 4 , a theory with a quadratic and quartic interaction is studied, and it is shown that it exhibits third order phase transition except for a specific value of the coupling constant. It is shown that for this value, the order of the transition is $5 / 2$. Finally, in section 5 the standard technique is used to investigate the critical behavior of this theory, and again it is checked that the order of the transition is $5 / 2$ at this specific value of the coupling constant.

\section{Typical gYM $\mathrm{gM}_{2}$ theories}

The partition function of the $\mathrm{gYM}_{2}$ on a sphere is 10 , 11

$$
Z=\sum_{r} d_{r}^{2} e^{-A \Lambda(r)},
$$

where $r$ 's label the irreducible representations of the gauge group, $d_{r}$ is the dimension of the representation $r, A$ is the area of the sphere, and $\Lambda(r)$ is :

$$
\Lambda(r)=\sum_{k=1}^{p} \frac{a_{k}}{N^{k-1}} C_{k}(r),
$$

in which $C_{k}$ is the $k^{\prime}$ th Casimir of group, and $a_{k}$ 's are arbitrary constants. Now consider the gauge group $\mathrm{U}(N)$ and parameterize its representation by the integers $n_{1} \geq n_{2} \geq \cdots \geq n_{N}$. It is found that

$$
\begin{aligned}
d_{r} & =\prod_{1 \leq i<j \leq N}\left(1+\frac{n_{i}-n_{j}}{j-i}\right), \\
C_{k} & =\sum_{i=1}^{N}\left[\left(n_{i}+N-i\right)^{k}-(N-i)^{k}\right] .
\end{aligned}
$$

For the partition function (1) to be convergent, it is necessary that $p$ in (2) be even and $a_{p}$ be positive. 
In the large $-N$ limit, the above summation is replaced by a path integration over the continuous function

$$
\phi(x):=-n(x)-1+x,
$$

where

$$
0 \leq x:=i / N \leq 1 \quad \text { and } \quad n(x):=n_{i} / N .
$$

The partition function (11) then becomes

$$
Z=\int \prod_{0 \leq x \leq 1} d \phi(x) e^{S[\phi(x)]}
$$

where

$$
S(\phi)=N^{2}\left\{-A \int_{0}^{1} d x G[\phi(x)]+\int_{0}^{1} d x \int_{0}^{1} d y \log |\phi(x)-\phi(y)|\right\},
$$

apart from an unimportant constant, and

$$
G(\phi)=\sum_{k=1}^{p}(-1)^{k} a_{k} \phi^{k}
$$

Introducing the density function

$$
\rho[\phi(x)]=\frac{d x}{d \phi(x)}
$$

it is seen that it satisfies

$$
\int_{b}^{a} \rho(z) d z=1
$$

where $[b, a]$ is the interval corresponding to the values of $\phi$. If $G(\phi)$, and therefore $\rho(z)$, is even, then $b=-a$. The condition $n_{1} \geq n_{2} \geq \cdots \geq n_{N}$ demands

$$
\rho(z) \leq 1
$$

As $N \rightarrow \infty$, only that representation contributes to the partition function (6) which maximizes $S$. This representation is found to be specified by the solution of the following integral equation 17.

$$
g(z)=\mathrm{P} \int_{b}^{a} \frac{\rho\left(z^{\prime}\right) d z^{\prime}}{z-z^{\prime}}
$$

where $\mathrm{P}$ indicates the principal value of the integral, and

$$
g(z):=\frac{A}{2} G^{\prime}(z)
$$


The free energy of the theory is defined through

$$
F:=-\frac{1}{N^{2}} \ln Z
$$

from which we have

$$
F^{\prime}(A)=\int_{b}^{a} d z G(z) \rho(z) .
$$

Using the standard method of solving the integral equation (12), the density function $\rho(z)$ is obtained in terms of the two parameters $a$ and $b$, where $a$ and $b$ themselves must be calculated by solving two other equations [17. If $G$ is even, the results are

$$
\rho(z)=\frac{\sqrt{a^{2}-z^{2}}}{\pi} \sum_{n, q=0}^{\infty} \frac{(2 n-1) ! !}{2^{n} n !(2 n+q+1) !} a^{2 n} z^{q} g^{(2 n+q+1)}(0),
$$

and

$$
\sum_{n=0}^{\infty} \frac{(2 n-1) ! !}{2^{n} n !(2 n-1) !} a^{2 n} g^{(2 n-1)}(0)=1
$$

where $g^{(n)}$ is the $n$th derivative of $g(z)$. As it is seen from (17), one cannot find the closed form of $a$ for arbitrary gYM 2 's (that is, for arbitrary $G$ 's).

The function $\rho(z)$ found from (16), obviously depends on the area $A$. Its absolute maximum is an increasing function of $A$. But the constraint (11) tells that this maximum must not exceed 1. So, the function $\rho(z)$ obtained from (16) is valid only for $A$ less than some critical value $A_{c}$. $A_{c}$ is the value of $A$ at which the maximum of $\rho$ becomes 1 . The region $A<A_{c}$ is called the weak-coupling region. In the region $A>A_{c}$ (the strong-coupling region), the following ansatz for $\rho(z)$ is used [17].

$$
\rho_{s}(z)= \begin{cases}1, & z \in L^{\prime} \\ \tilde{\rho}_{s}(z), & z \in L,\end{cases}
$$

where $L^{\prime}$ is some interval containing the point (or points) maximizing $\rho$. The form of $\rho_{s}$ is usually found to consist of some integrals not expressible in terms of elementary functions. In special cases, it has been calculated perturbatively for areas slightly more than the critical area (14, 15, 17, 18] for example). Having obtained $\rho_{s}$, the difference between the free energies in strong and weak regimes $\left(F_{s}-F_{w}\right)$, for areas slightly more than the critical area is calculated to obtain the order of the transition.

Consider this same problem of phase transition from a different point of view. Denote by $\rho_{w}(z)$ the weak-region density function, and by $\rho_{s}(z)$ the density function (18). Suppose that $\rho_{w}(z)$ attains its (absolute) maximum at $z=z_{0}$. We have

$$
\rho_{w}(z)=\rho_{w}\left(z_{0}\right)+\frac{1}{2} \rho_{w}^{\prime \prime}\left(z_{0}\right)\left(z-z_{0}\right)^{2}+\cdots
$$


in which $\rho_{w}^{\prime \prime}\left(z_{0}\right)$ is assumed to be nonzero. For $A$ slightly more than $A_{c}, \rho_{w}\left(z_{0}\right)$ is slightly more than 1 . We want to find the points $z_{1}$ satisfying $\rho_{w}\left(z_{1}\right)=1$. It is easy to see that

$$
\left|z_{1}-z_{0}\right| \approx \sqrt{2\left[1-\rho_{w}\left(z_{0}\right)\right] / \rho_{w}^{\prime \prime}\left(z_{0}\right)} \sim \alpha^{1 / 2}
$$

where $\alpha:=\rho_{w}\left(z_{0}\right)-1$. Next, consider $F_{s}-F_{w}$ :

$$
\begin{aligned}
F\left[\rho_{s}\right]-F\left[\rho_{w}\right]= & \int\left[\frac{\delta F}{\delta \rho(z)}\right]_{\rho_{w}}\left[\rho_{s}(z)-\rho_{w}(z)\right] d z \\
& +\frac{1}{2} \int\left[\frac{\delta^{2} F}{\delta \rho(z) \delta \rho\left(z^{\prime}\right)}\right]_{\rho_{w}}\left[\rho_{s}(z)-\rho_{w}(z)\right] \\
& \times\left[\rho_{s}\left(z^{\prime}\right)-\rho_{w}\left(z^{\prime}\right)\right] d z d z^{\prime}+\cdots
\end{aligned}
$$

As $\rho_{w}$ is the classical solution, it satisfies

$$
\left(\frac{\delta S}{\delta \rho}\right)_{\rho_{w}}=\lambda
$$

where $\lambda$ is the Lagrange--multiplier used to introduce the condition (10) in the action $S$. So the first term of the right-hand side of (21) becomes

$$
\begin{aligned}
\int\left[\frac{\delta F}{\delta \rho(z)}\right]_{\rho_{w}}\left[\rho_{s}(z)-\rho_{w}(z)\right] d z & =-\frac{\lambda}{N^{2}}\left[\int \rho_{s}(z) d z-\int \rho_{w}(z) d z\right] \\
& =0
\end{aligned}
$$

in which we have used the fact that $\rho_{w}$ and $\rho_{s}$, both satisfy (10) - perhaps with different integration regions, but it is not important, as one may define $\rho$ to be zero outside the integration region and then extend the integration region to the whole real line. Next, consider the second term of (21). One can divide the integration region into three parts:

$R_{1}: z, z^{\prime} \in L_{s}$

$R_{2}: z, z^{\prime} \in L_{w}$,

$R_{3}: z \in L_{s}$ and $z^{\prime} \in L_{w}$ or visa versa,

where $L_{s}$ is the region in which $\rho_{w}>1$, and $L_{w}$ is the remaining region. We have

$$
\rho_{w}(z)-\rho_{s}(z) \sim \alpha, \quad z \in L_{s} .
$$

From this, it is seen that

$$
\int_{L_{w}}\left[\rho_{w}(z)-\rho_{s}(z)\right] d z \sim \alpha^{3 / 2}
$$

as the width of the region $L_{s}$ is of the order $\alpha^{1 / 2}(20)$. Using (10) for $\rho_{s}$ and $\rho_{w}$, it is seen that

$$
\int_{L_{w}}\left[\rho_{s}(z)-\rho_{w}(z)\right] d z \sim \alpha^{3 / 2}
$$


The width of $L_{w}$ is of the order $\alpha^{0}$. This shows that on the average,

$$
\rho_{s}(z)-\rho_{w}(z) \sim \alpha^{3 / 2}, \quad z \in L_{w} .
$$

Using these, it is seen that in $R_{1}$, the integrand in the second term of (21) is of the order $\alpha^{2}$, while the area of the integration region is of the order $\alpha$. So the integral in this region is of the order $\alpha^{3}$. In $R_{2}$, the integrand is of the order $\alpha \times \alpha^{3 / 2}$, and the area of $R_{2}$ is of the order $\alpha^{1 / 2}$. So the integral in this region too is of the order $\alpha^{3}$. Finally, in $R_{3}$, the integrand is of the order $\alpha^{3 / 2} \times \alpha^{3 / 2}$, while th area of $R_{3}$ is of the order $\alpha^{0}$. So, the integral in this region is of the order $\alpha^{3}$ as well. One therefor deduces

$$
F_{s}-F_{w} \sim \alpha^{3} .
$$

Here we have used $\rho_{w}^{\prime \prime}\left(z_{0}\right) \neq 0$.

To find the critical behavior of the system, we must obtain $F_{s}-F_{w}$ as a function of $A-A_{c}$. To do so, consider $\alpha$ as a function of $A$ and expand it around $A=A_{c}$ :

$$
\alpha(A)=\alpha\left(A_{c}\right)+\alpha^{\prime}\left(A_{c}\right)\left(A-A_{c}\right)+\cdots .
$$

But $\alpha\left(A_{c}\right)=0$. So if $\alpha^{\prime}\left(A_{c}\right) \neq 0$, we have

$$
\alpha \sim\left(A-A_{c}\right),
$$

and from this

$$
F_{s}-F_{w} \sim\left(A-A_{c}\right)^{3},
$$

that is, the model has a third-order phase transition. What we have proved, is that if $\rho_{w}^{\prime \prime}\left(z_{0}\right) \neq 0$ and $\alpha^{\prime}\left(A_{c}\right) \neq 0$, then the order of the phase transition is 3 . In fact, to prove this we have made an implicit assumption that (27) holds not only on the average, but at almost every point in $L_{w}$. There might be very special cases where the difference between $\rho_{s}$ and $\rho_{w}$ is larger than $\alpha^{3}$ but changes sign exactly in a such a manner that the integral of this difference is of the order $\alpha^{3 / 2}$. Also note that the whole reasoning is independent of the number of points at them $\rho_{w}$ attains its absolute maximum.

Continuing to make this assumption, let's see what happens if either $\rho_{w}^{\prime \prime}\left(z_{0}\right)$ or $\alpha^{\prime}\left(A_{c}\right)$ is zero. As $z_{0}$ is the point which maximizes $\rho_{w}$, the first nonzero derivative of $\rho_{w}$ at this point should be an even derivative. Let it be the $(2 m)$ th derivative. Also, $\alpha$ should be increasing with respect to $A$ at $A_{c}$. This shows that its first nonzero derivative should be on odd derivative. Let it be the $(2 l-1)$-th derivative. It follows that the width of $L_{s}$ is of the order $\alpha^{1 /(2 m)}$, and from this

$$
F_{s}-F_{w} \sim \alpha^{2+(1 / m)} .
$$

One also has

$$
\alpha \sim\left(A-A_{c}\right)^{2 l-1} \text {. }
$$


Combining these two, one arrives at

$$
F_{s}-F_{w} \sim\left(A-A_{c}\right)^{(2 l-1)[2+(1 / m)]},
$$

where $m$ and $l$ are positive integers.

\section{$3 \quad G(\phi)=\phi^{2 k} \mathbf{g Y M}_{2}$ 's}

For $G(\phi)=\phi^{2 k}$ ( $k$ an integer greater than 1$)$, it has been shown that the density function $\rho_{w}(z),(16)$, has a minimum at $z=0$ and two symmetric maxima at points $z_{0}= \pm a_{k} \sqrt{y_{k}}$, [18]. $a_{k}$ is the parameter appearing in (16), and can be evaluated from (17):

$$
a_{k}=\left[\frac{2^{k}(k-1) !}{A(2 k-1) ! !}\right]^{\frac{1}{2 k}} .
$$

$y_{k}$ is independent of $a_{k}$ and is determined from

$$
-1+\sum_{n=0}^{k-2} \frac{(2 n-1) ! !}{2^{n+1}(n+1) !} y_{k}^{-(n+1)}=0 .
$$

Now it is possible to find the $A$-dependence of $\rho\left(z_{0}\right)$. Using (16),

$$
\rho_{w}(z)=\frac{k A}{\pi} \sqrt{a_{k}^{2}-z^{2}} \sum_{n=0}^{k-1} \frac{(2 n-1) ! !}{2^{n} n !} a_{k}^{2 n} z^{2 k-2 n-2} .
$$

From this,

$$
\begin{aligned}
\rho_{w}\left(z_{0}\right) & =A^{\frac{1}{2 k}} \frac{k \sqrt{1-y_{k}}}{\pi}\left[\frac{2^{k}(k-1) !}{(2 k-1) ! !}\right]^{\frac{2 k-1}{2 k}} \sum_{n=0}^{k-1} \frac{(2 n-1) ! !}{2^{n} n !} y_{k}^{k-n-1} \\
& =: \quad A^{\frac{1}{2 k}} f(k),
\end{aligned}
$$

where $f(k)$ is independent of $A$. $A_{c}$ is determined by $\rho_{k}\left(z_{0}\right)=1$, which yields

$$
A_{c}=\frac{1}{[f(k)]^{2 k}} \text {. }
$$

From this,

$$
\alpha(A)=\left(\frac{A}{A_{c}}\right)^{\frac{1}{2 k}}-1,
$$

which shows that the derivative of $\alpha$ with respect to $A$ never vanishes. It is also easy to check that the $\rho_{w}^{\prime \prime}\left(z_{0}\right)$ doesn't vanish either. Using $\rho_{w}^{\prime}\left(z_{0}\right)=0$, it is seen that

$\rho_{w}^{\prime \prime}\left(z_{0}\right)=\frac{k A a^{2 k-3}}{\pi} \frac{(2 k-1) u}{\sqrt{1-u^{2}}}\left[-(2 k-1) u^{2 k-3}+\sum_{n=0}^{k-2} \frac{(2 n-1) ! !}{2^{n+1}(n+1) !} u^{2 k-2 n-5}\right]$ 


$$
=-\frac{k A a^{2 k-3}}{\pi} \frac{2 k-1}{\sqrt{1-u^{2}}} \sum_{n=0}^{k-2} \frac{(2 n-1) ! !}{2^{n} n !} u^{2 k-2 n-4},
$$

which is clearly negative. Here

$$
u:=\frac{z_{0}}{a},
$$

and (36) has been used. We conclude all $\mathrm{gYM}_{2}$ 's with $G(\phi)=\phi^{2 k} \mathrm{gYM}_{2}$ 's exhibit third-order phase transition.

\section{The model $G(\phi)=\phi^{2}+g \phi^{4}$}

We now consider $G(\phi)=\phi^{2}+g \phi^{4}$, with $g>0$ so that in this $G$ has a minimum. Using (16), we have

$$
\rho_{w}(z)=\frac{A}{\pi} \sqrt{a^{2}-z^{2}}\left(1+g a^{2}+2 g z^{2}\right),
$$

and from (17),

$$
\frac{1}{2} A a^{2}+\frac{3}{4} g A a^{4}=1
$$

The shape of $\rho_{w}(z)$ depends on $A$. For $3 g a^{2}<1$, or $A>4 g$ using (44), $\rho_{w}(z)$ has only one maximum at $z=0$. For $A<4 g$, it has a minimum at $z=0$ and two maxima at $\pm z_{0}$. We consider three distinct cases.

\section{1 $g<A_{c} / 4$}

In this case, $\rho_{w}$ has two maxima for small areas. However, if the area exceeds $4 g<A_{c}, \rho_{w}$ will have only one maximum. As this happens before the critical area, the phase transition properties are governed by a one-maximum density function. The maximum of this function occurs at $z=0$. Evaluating $a$ from (44), we arrive at

$$
\rho_{w}(0)=\frac{A}{2 \pi}\left(2+\sqrt{1+12 \frac{g}{A}}\right) \sqrt{\frac{3}{g}\left(-1+\sqrt{1+12 \frac{g}{A}}\right)},
$$

and from this

$$
\alpha^{\prime}\left(A_{c}\right)=\frac{1}{\sqrt{27 \pi^{2} g}} \frac{\left(3 g / A_{c}\right)-1+\sqrt{1+12 g / A_{c}}}{\sqrt{-1+\sqrt{1+12 g / A_{c}}}}>0 .
$$

It is also seen that

$$
\rho_{w}^{\prime \prime}(0)=\frac{A}{\pi a}\left(3 g a^{2}-1\right)<0
$$

So the order of the transition is 3 . 


\section{2 $g>A_{c} / 4$}

In this case, for areas up to $4 g>A_{c}$, the density function has two maxima at $\pm z_{0}$ :

$$
z_{0}=\sqrt{\frac{1}{6 g}\left(-2+\sqrt{1+12 \frac{g}{A}}\right)},
$$

From this, one obtains $\rho_{w}\left(z_{0}\right)$, and then

$$
\alpha^{\prime}\left(A_{c}\right)=\sqrt{\frac{2}{27 g \pi^{2}}} \frac{1+3 g / A_{c}}{\left(1+12 g / A_{c}\right)^{1 / 4}}>0 .
$$

It is also not difficult to check that $\rho_{w}^{\prime \prime}\left(z_{0}\right)$ is negative. So in this case too, the order of the phase-transition is 3 .

\section{3 $g=A_{c} / 4$}

In this case, for $A<4 g=A_{c}$ the function $\rho_{w}(z)$ has two maxima at $\pm z_{0}$ and one minimum at $z=0$. As $A$ is increased, the difference $\rho_{w}\left(z_{0}\right)-\rho_{w}(0)$ becomes smaller, and at $A=A_{c}$ it becomes zero. For $A>A_{c}, \rho_{w}(z)$ has only one maximum at $z=0$. At $A=A_{c}$, the maximum of the density function becomes 1 (and occurs at $z=0$ ). Using this, the form of $\rho_{w}$ from (43), and (44), it is seen that

$$
\begin{gathered}
A_{c}=\frac{27}{64} \pi^{2}, \\
g_{0}=\frac{A_{c}}{4}=\frac{27}{256} \pi^{2},
\end{gathered}
$$

and

$$
a_{c}=\frac{16}{9 \pi} \text {. }
$$

The interesting point for $g=g_{0}$ is that $\left.\rho_{w}^{\prime \prime}(0)\right|_{A=A_{c}}=0$. In fact, one can see that

$$
\begin{aligned}
\rho_{w}^{\prime \prime}(0) & =\frac{3 g_{0} A a\left(a+a_{c}\right)}{\pi}\left(a-a_{c}\right) \\
& =-\frac{4}{3 \pi}\left(A-A_{c}\right)+\cdots,
\end{aligned}
$$

while $\rho_{w}^{(4)}(0)$ is negative. So for $z_{1}$, satisfying $\rho_{w}\left(z_{1}\right)=1$, or

$$
\frac{1}{2 !} \rho_{w}^{\prime \prime}(0) z_{1}^{2}+\frac{1}{4 !} \rho_{w}^{(4)}(0) z_{1}^{4}=1-\rho_{w}(0),
$$

one obtains

$$
\left|z_{1}\right| \sim \alpha^{1 / 4}
$$


One also has

$$
\alpha=\frac{28}{27 \pi^{2}}\left(A-A_{c}\right)+\cdots,
$$

from which it is seen that

$$
\left|z_{1}\right| \sim\left(A-A_{c}\right)^{1 / 4}
$$

This is an example of the case $m=2$ and $l=1$, discussed at the end of section 2. It follows then that

$$
F_{s}-F_{w} \sim\left(A-A_{c}\right)^{5 / 2} .
$$

The model $G(\phi)=\phi^{2}+\left(27 \pi^{2} / 64\right) \phi^{4}$ exhibits thus a phase transition of order $5 / 2$.

\section{Explicit evaluation of the order of the phase transition of the model $G(\phi)=\phi^{2}+\frac{27 \pi^{2}}{64} \phi^{4}$}

We now try to study the phase transition of the model $G(\phi)=\phi^{2}+g_{0} \phi^{4}$ explicitly $\left(g_{0}=27 \pi^{2} / 64\right)$. Using (15) and (43), one finds that in the weak region

$$
F_{w}^{\prime}(A)=\frac{1}{8} a^{4} A+\frac{5}{16} g_{0} a^{6} A+\frac{9}{64} g_{0}^{2} a^{8} A,
$$

where $a$ can be found from (44). In the strong region, one uses the following ansatz for $\rho_{s}(z)$

$$
\rho_{s}(z)= \begin{cases}1, & z \in[-b, b] \\ \tilde{\rho}_{s}(z), & z \in[-a,-b] \bigcup[b, a],\end{cases}
$$

(as $\rho_{w}(z)$ has only one maximum for $A>A_{c}$ ). Using the standard method of solving the integral equations obtained (see [18] for more details) one arrives at

$$
\begin{aligned}
F_{s}^{\prime}(A)= & \left(\frac{1}{8} M^{2}-\frac{1}{2} N\right) \int_{-b}^{b} \frac{d \lambda}{U(\lambda)}+\frac{1}{2} M \int_{-b}^{b} \frac{\lambda^{2} d \lambda}{U(\lambda)}-\int_{-b}^{b} \frac{\lambda^{4} d \lambda}{U(\lambda)} \\
& +g_{0}\left[\left(-\frac{1}{4} M N+\frac{1}{16} M^{3}\right) \int_{-b}^{b} \frac{d \lambda}{U(\lambda)}\right. \\
& +\left(\frac{1}{8} M^{2}-\frac{1}{2} N\right) \int_{-b}^{b} \frac{\lambda^{2} d \lambda}{U(\lambda)} \\
& \left.+\frac{1}{2} M \int_{-b}^{b} \frac{\lambda^{4} d \lambda}{U(\lambda)}-\int_{-b}^{b} \frac{\lambda^{6} d \lambda}{U(\lambda)}\right] \\
& -2 g_{0} A\left(\frac{1}{4} M N-\frac{1}{16} M^{3}\right) \\
& -2 g_{0}^{2} A\left(-\frac{1}{8} N^{2}+\frac{3}{16} M^{2} N-\frac{5}{128} M^{4}\right),
\end{aligned}
$$


in which

$$
\begin{aligned}
U(\lambda) & :=\sqrt{\left(a^{2}-\lambda^{2}\right)\left(b^{2}-\lambda^{2}\right)} \\
M & :=a^{2}+b^{2} \\
N & :=a^{2} b^{2} .
\end{aligned}
$$

The parameters $a$ and $b$ are determined from following relations.

$$
A+g_{0} A M-\int_{-b}^{b} \frac{d \lambda}{U(\lambda)}=0
$$

and

$$
\frac{1}{2} M A+g_{0} A\left(\frac{3}{4} M^{2}-N\right)-\int_{-b}^{b} \frac{\lambda^{2} d \lambda}{U(\lambda)}=1 .
$$

As we are interested in phase transition, it is sufficient to look at the solutions for $A$ slightly greater than $A_{c}$, which we obtain perturbatively.

To do so, we use the following change of variables

$$
a=: a_{c}(1-h),
$$

and

$$
(b / a)^{2}=: v,
$$

in which $a_{c}$ is given by (52). Eliminating $A$ from (63) and (64), and solving the result for $h$, one obtains

$$
h=\frac{27}{224} v^{2}+\frac{131}{6272} v^{3}+\cdots,
$$

from this, and (63), one obtains $A$ in terms of $v$ :

$$
\frac{A-A_{c}}{A_{c}}=\frac{9}{28} v^{2}+\frac{109}{784} v^{3}+\cdots .
$$

Note that $A-A_{c}$ is proportional to $b^{4}$ (in the leading order), as expected. Substituting these in (61), one obtains $F_{s}^{\prime}$ :

$$
F_{s}^{\prime}=\frac{1}{35721 \pi^{2}}\left(36848-10836 v^{2}+6289 v^{3}+\cdots\right) .
$$

From (44), one obtains $a$ in terms of $A$. Substituting this in (59), and using (68) as a definition for $v$, one obtains

$$
F_{w}^{\prime}=\frac{1}{35721 \pi^{2}}\left(36848-10836 v^{2}-4687 v^{3}+\cdots\right),
$$

from which

$$
F_{s}^{\prime}(A)-F_{w}^{\prime}(A)=\frac{224}{729 \pi^{2}} v^{3}+\cdots
$$


Using (68), one can write this as

$$
F_{s}^{\prime}(A)-F_{w}^{\prime}(A)=\frac{196 \sqrt{7}}{729 A_{c}}\left(\frac{A-A_{c}}{A_{c}}\right)^{3 / 2}+\cdots .
$$

which proves that the order of the phase transition is $5 / 2$, in agreement with (58).

\section{Acknowledgement}

M. Alimohammadi would like to thank the research council of the University of Tehran, for partial financial support. The authors would also like to thank Institute for Studies in Theoretical Physics and Mathematics for partial financial support.

\section{References}

[1] D. J. Gross; Nucl. Phys. B400 (1993) 161.

[2] J. Minahan; Phys. Rev. D47 (1993) 3430.

[3] D. J. Gross \& W. Taylor; Nucl. Phys. B400 (1993) 181.

[4] D. J. Gross \& W. Taylor; Nucl. Phys. B400 (1993) 395.

[5] D. Fine; Commun. Math. Phys. 134 (1990) 273; 140 (1991) 321.

[6] E. Witten; Commun. Math. Phys. 141 (1991) 153.

[7] M. Blau \& G. Thompson; Int. J. Mod. Phys. A7 (1992) 3781.

[8] E. Witten; J. Geom. Phys. 9 (1992) 303.

[9] M. R. Douglas, K. Li, \& M. Staudacher; Nucl. Phys. B240 (1994) 140.

[10] O. Ganor, J. Sonnenschein, \& S. Yankielowicz; Nucl. Phys. B434 (1995) 139.

[11] M. Khorrami \& M. Alimohammadi; Mod. Phys. Lett. A12 (1997) 2265.

[12] M. Alimohammadi \& M. Khorrami; Int. J. Mod. Phys. A12 (1997) 1959.

[13] M. Alimohammadi \& M. Khorrami; Z. Phys. C76 (1997) 729.

[14] M. R. Douglas \& V. A. Kazakov; Phys. Lett. B319 (1993) 219. 
[15] A. Aghamohammadi, M. Alimohammadi, \& M. Khorrami; Mod. Phys. Lett. A14 (1999) 751.

[16] B. Rusakov \& S. Yankielowicz; Phys. Lett. B339 (1994) 258.

[17] M. Alimohammadi, M. Khorrami, \& A. Aghamohammadi; Nucl. Phys. B510 (1998) 313.

[18] M. Alimohammadi \& A. Tofighi; Eur. Phys. J. C8 (1999) 711. 